\title{
Re: Perineural Invasion by Transitional Cell Carcinoma of the Bladder in Patients submitted to Radical Cystectomy: What is the Prognostic Value?
}

\author{
Alberto A Antunes, Luciano J. Nesrallah, Marcos F. Dall'Oglio, Alexandre Crippa, Adriano J. \\ Nesrallah, Mario Paranhos, Katia R. Leite, Miguel Srougi \\ Division of Urology, University of Sao Paulo Medical School, Sao Paulo, SP, Brazil and Laboratory \\ of Surgical and Molecular Pathology, Syrian Lebanese Hospital, Sao Paulo, Brazil
}

Int Braz J Urol, 33: 161-166, 2007

To the Editor:

The paper by Antunes et al. is an excellent contribution to a controversial issue: the importance of perineural invasion as a prognostic factor for bladder cancer after radical cystectomy. The relationship between perineural invasion and prognosis has been demonstrated to be poor in a number of malignancies. Particularly in the prostate, perineural invasion may have importance as a predictor of extraprostatic extension. In the bladder, controversy exists on whether the pathologic features of vascular (blood and/or lymphatic), and perineural invasion have any role as prognostic indicators. In a study cited by Antunes et al., on univariate analysis lymphatic, blood vessel and perinerual tumor invasion showed strong prognostic significance. However, on multivariate analysis only blood vessel invasion, invasion depth and regional lymph node status were independent prognostic factors (1). In another paper also cited by Antunes et al., univariate analysis revealed that vas- cular invasion, lymphatic invasion, and perineural invasion were significant prognostic predictors of overall survival. However, only the tumor stage and vascular invasion proved to be independent prognostic predictors of disease-specific survival on multivariate analysis (2). Independent of the controversy, in my opinion vascular and perineural invasion in vesical cancer should be commented on the pathology report.

\section{References}

1. Leissner J, Koeppen C, Wolf HK: Prognostic significance of vascular and perineural invasion in urothelial bladder cancer treated with radical cystectomy. J Urol. 2003; 169: 955-60.

2. Hong SK, Kwak C, Jeon HG, Lee E, Lee SE: Do vascular, lymphatic, and perineural invasion have prognostic implications for bladder cancer after radical cystectomy? Urology. 2005; 65: 697-702.

Dr. Athanase Billis Full-Professor of Pathology State University of Campinas, Unicamp Campinas, Sao Paulo, Brazil E-mail:athanase@fcm.unicamp.br 